\title{
Situación actual de la economía japonesa y las reformas de Koizumi
}

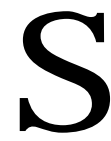

egún el reconocido economista japonés Nakatani Iwao, hay cinco aspectos clave en el deterioro actual de la economía japonesa: la gran deflación en la economía real y los créditos de difícil recuperación de los bancos; el grave deterioro de las economías regionales: el estancamiento del sistema empresarial; la disminución del gasto por parte de los consumidores y la poca eficacia y transparencia en la política del gobierno.

Como consecuencia de las convulsiones del sistema financiero, cada año después del colapso de la "economía de burbuja" de principio de la década de los noventa, el valor de los activos se ha ido reduciendo a un ritmo de más de 100 millones de millones de yenes anuales. Así, como resultado del derrumbe de los precios de las acciones y de las propiedades que actuaban como garantía de sus créditos, los bancos japoneses han acumulado préstamos en mora por un monto de más de 1 millón de millones de dólares (25\% del PIB), de los cuales el $30 \%$ son directamente incobrables.

Este derrumbe ha tenido graves repercusiones: algunos grandes bancos han quebrado y muchos otros han sido estatizados para ser "saneados" y luego reprivatizados. La deuda total de las compañías bancarias y financieras ha venido en aumento, así también el número de quiebras del sector corporativo, que en abril de 2003 alcanzaba la cifra de 1514 quiebras empresariales, principalmente motivadas por las caídas de las ventas (bajos precios y poco consumo), el estancamiento industrial y las dificultades en la recuperación de los créditos. Pero el caso del sistema bancario es mucho más grave, ya que los bancos japoneses tienen inflados los valores de las

\footnotetext{
* Investigador del Centro de Investigaciones de la Economía Mundial (CIEM), Cuba.
}

propiedades que han recibido en garantía en más del doble de su valor de mercado. ${ }^{1}$

En el caso de las familias japonesas, debido a la disminución en un $70 \%$ del valor de las casas adquiridas mediante créditos hipotecarios, se anuncian pérdidas en el orden de 250 mil millones de dólares. A pesar de las bajas tasa de interés, en la actualidad las hipotecas absorben ardedor del $8 \%$ del ingreso personal disponible.

Una de las medidas que todavía se está aplicando, y que no acaba de resolver el problema, fue la reducción sustancial de las tasas de interés para permitir que los deudores morosos reestructuraran sus obligaciones y para impulsar el crédito al consumo. La baja de las tasas de interés alcanzó el récord histórico de cero, para los préstamos del Banco de Japón (Banco Central) al sistema bancario. Pero como esto no ha bastado, el gobierno ha inyectado aproximadamente 67 millones de dólares a los quince mayores bancos para mejorar sus balances. Además, ha financiado la fusión de toda una serie de bancos e instituciones problemáticas y otras quebradas fueron directamente estatizadas.

A pesar de estas medidas, la situación de los grandes bancos sigue prácticamente igual, ya que no tienen a quien otorgarle préstamos porque el país todavía está lleno de empresas endeudadas y demasiado débiles para invertir en el futuro. El hiperendeudamiento, el exceso de la capacidad instalada, la extrema debilidad de los mercados y los nulos beneficios explican que la demanda de crédito bancario por parte de las empresas haya registrado su mayor declinación en toda las historia después de la segunda guerra mundial. 
Uno de los hechos relevantes en 1999 , como muestra de este caos económico, es la quiebra de dos importantes bancos regionales, el Kokumin Bank y el Namihaya Bank. Este último es significativo porque se trata de un banco creado con la fusión, financiada por el gobierno, de otros dos bancos virtualmente quebrados. La crisis de la banca regional, que hasta el momento había estado en un segundo plano, ya se convertiría en algo muy preocupante para el gobierno nipón. ${ }^{2}$

Con respecto al problema de las autonomía de las regiones de Japón, es importante señalar que el gobierno central es quien distribuye alrededor del $70 \%$ de los ingresos fiscales que recauda de las administraciones locales y utiliza su poder fiscal para mantenerlas controladas. En este sentido, las necesidades locales reales no tienen probabilidades de ser aprobadas por el gobierno. Éste se limita a dar el visto bueno a solicitudes de tipo estándar y generalizadas, sin tomar en cuenta las particularidades locales.

Con el fin de solucionar esta problemática y para acelerar la reforma estructural, se aprobó en 2003 la creación de 57 zonas económicas especiales, que están sujetas a un trato preferencial de liberalización, incluidas ocho zonas en las que las empresas privadas podrán emprender actividades agrícolas.

Para revitalizar y dar más independencia a las regiones, se pretende lograr un desarrollo basado en las especificidades de las regiones, para que puedan cubrir con sus propios fondos sus necesidades de la administración local, asegurando el ingreso de los impuestos locales.

La zona especial se deja en manos únicamente de la iniciativa de los municipios y debe propiciar la competencia entre las regiones. Después de la aprobación de la asamblea local, cada municipio solicita al gobierno central la exención de la regulación que impide participar en las nuevas actividades, y de esta forma se comienza a formar "las zonas especiales" Según el secretariado de Estado encargado de las zonas especiales, Yoshitada Konoike, las provincias y municipios han enviado peticiones al gobierno para establecer otras 129 zonas económicas especiales. Dichas zonas son las primeras de desregulación que tiene Japón y forman parte de las reformas estructurales del primer ministro Junichiro Koizumi. ${ }^{3}$

El entorno económico desfavorable ha limitado la inversión en proyectos nuevos. El famoso triángulo de acero (políticos, burócratas y empresarios), que fue la base del sistema de toma de decisiones durante la época de crecimiento acelerado, actualmente está en crisis: sólo hay que observar la gran diferencia entre burócratas y empresarios. Corregir esta situación implica restablecer un sistema de incentivos que premie el esfuerzo emprendedor del empresariado japonés.

El cuarto problema que enfrenta la economía japonesa actualmente radica en la falta de incentivos para el consumo personal. Esto se debe principalmente a la inseguridad laboral que afecta a Japón desde principio de los años noventa. Pese al fuerte descenso generalizado y continuo de los precios (deflación), la desconfianza mantiene el consumo estancado, y es que la deflación reduce los beneficios de las empresas, estimula el desempleo, la disminución de los salarios y agrava las deudas de los bancos.

La inseguridad en el ingreso laboral y el desempleo han desestimulado el consumo y 
aumentado el ahorro motivo-precaución. Este problema de la disminución de la demanda interna ha traído problemas serios a la economía nipona, ya que el consumo personal representa el $60 \%$ del producto interno bruto japonés, que es clave para la salud económica del país.

Las encuestas revelan que el pueblo japonés se siente más pesimista con respecto a su futuro que en cualquier otro momento desde la segunda guerra mundial. Los nipones temen al retiro, les preocupa perder sus trabajos y no creen en las promesas del gobierno. Los ancianos cuidan celosamente sus ahorros; las familias con niños están reduciendo sus gastos e incluso los "solteros parásitos" - jóvenes que disponen de sueldos generosos para gastar y aún viven con sus padres- están comenzando a cambiar sus costumbres derrochadoras. Según dicen algunos especialistas "la probabilidad de recuperación de la demanda doméstica es casi nula". La única manera de recuperar el consumo es aumentar los ingresos y no hay fórmula mágica para lograrlo.

En el segundo semestre de 2003 las cifras revelaron que el gasto de los consumidores avanzó apenas $0.3 \%$ respecto al trimestre anterior. En este sentido, los expertos manifestaron que muchos consumidores acudieron a sus ahorros y advirtieron que el alza en el consumo es imposible mientras la tasa de desempleo oscile en torno al 5\%, nivel que mantiene desde hace 20 meses. ${ }^{4}$ La tasa de desempleo llegó a alcanzar el récord de 5.5\% durante tres meses consecutivos en 2003, y actualmente está en 5.4\%. Hoy existen alrededor de 3,57 millones de personas desempleadas, en un país donde hasta hace poco se aseguraba el empleo de por vida.

Los japoneses tienden a ahorrar cada vez más de lo que tradicionalmente lo han hecho, lo cual ha limitado de manera significativa la capacidad del mercado interno para impulsar la demanda. Muchas personas ahorran por temor a quedarse sin empleo y no existe una seguridad social estable.
La alta propensión al ahorro debe interpretarse como un síntoma de desesperanza en algunas de las economías asiáticas. La incertidumbre con respecto al futuro ha elevado el nivel de ahorro de Japón, pero también contribuye a la deflación en ese país. Obviamente, un incremento en el ahorro significa que hay menor demanda. Esto hace que las empresas se encuentren sosteniendo grandes inversiones y obteniendo bajas ganancias, mientras recurren al recorte de precios necesario para deshacerse de los excedentes de stock. Al mismo tiempo, hay una reducción en el empleo que induce a los compradores a ahorrar más y gastar menos. Según se dice "los norteamericanos consumen más de lo que producen, y los japoneses producen más de lo que consumen"; la desaceleración de la demanda en este país no va a invertir esa tendencia.

El deterioro de la demanda interna, el enfriamiento de las exportaciones de Estados Unidos, la debilidad de las economías asiáticas, la volatilidad de los mercados bursátiles y sobre todo las dificultades del sector bancario y financiero, constituyen las causas fundamentales del estancamiento económico japonés.

El quinto y último problema es la ineficiencia y la opacidad del sector público: el pueblo nipón ha perdido la confianza en la política económica del gobierno para mejorar la situación económica. Las promesas incumplidas por parte de los primeros ministros, la ineficiencia en la aplicación de las medidas anunciadas y la inestabilidad política han influido en la pérdida de confianza en el gobierno. Durante los últimos trece años, Japón ha tenido once primeros ministros. Las encuestas realizadas apuntan que un alto porcentaje de la población no cree ni en los políticos, ni en los partidos; mientras los escándalos de corrupción que se han presentado recientemente contribuyen a esa percepción.

Los sucesivos gobiernos japoneses desperdiciaron toda la década de los noventa buscando la vía para sacar al país del estancamiento. Uno tras otro, adoptaron "once 
paquetes de estimulo fiscal" (aumento del gasto público, incluido el gasto armamentista y reducciones de impuestos), por un monto de 5 millones de millones de dólares, una cifra superior al Рів japonés (4,5 millones de millones de dólares). Al fracaso de cada uno de estos paquetes para sacar adelante la economía, le sucedía uno de mayor envergadura.

En el curso de la década de los noventa, como consecuencia de la inyección de fondos públicos, la deuda japonesa creció hasta alcanzar los 6 millones de millones de dólares, equivalente al $120 \%$ del PIB a finales de 1999 . La fenomenal inyección de gasto público no logró sacar al país de la recesión porque las empresas sobreendeudadas y con una enorme capacidad instalada excedente, no necesitaron aumentar sus inversiones. La mayoría de las empresas del estado ya están sobreendeudadas y enfrentan obstáculos insuperables para pagar los intereses de esos créditos, por lo tanto temen endeudarse cada vez más.

Las reducciones impositivas no lograron aumentar el consumo privado porque las familias no aumentaron sus ingresos efectivos, como consecuencia de la generalizada caída de los ingresos asalariados (por el aumento del desempleo y la reducción de los salarios nominales).

Para el economista estadounidense Ronald McKinnon, profesor de economía internacional de la Universidad de Standford, el prolongado estancamiento de la economía japonesa se ha convertido en "el gran fracaso de la macroeconomía moderna". Muchos analistas coinciden con esta afirmación. A pesar de aplicarse todas las políticas económicas de posguerra, éstas no han funcionado en Japón.

En los últimos años después de la explosión de la burbuja económica, la economía nipona se ha caracterizado por una vorágine de precios de activos en caída, crisis bancarias, beneficios empresarios reducidos y aumento de la deuda del estado.
Un ejemplo de poca o nula gestión realizada por el gobierno para tratar de frenar una recaída en los índices macroeconómicos es el caso del impopular ex primer ministro japonés, Yoshiro Mori, que tuvo que renunciar a su cargo a principios de 2001, después de haber estado solamente un año en el cargo, debido a una serie de escándalos, falta de transparencia e iniciativas para levantar la economía, lo que resultó en una crisis generalizada de credibilidad hacia el gobierno.

El nuevo primer ministro Junichiro Koizumi, quien sustituyó a Mori en abril de 2001, a diferencia de la pasividad de sus antecesores cuenta con una popularidad exclusiva; es miembro del Partido Liberal Demócrata y se ha comprometido a sacar a Japón adelante con una serie de reformas estructurales, fiscales y económicas.

Tras los intentos infructuosos de numerosos primeros ministros para sacar a Japón de la peor crisis de la posguerra, los japoneses parecen estar dispuestos a dar una oportunidad a un político que no promete seguridad y buena vida, sino que admite que las reformas necesarias para la reactivar la economía tienen un precio.

\section{El nuevo gobierno y las reformas de Koizumi}

El 21 de junio de 2001 el Consejo del Gobierno presidido por Koizumi decidió oficialmente las directrices básicas para las reformas estructurales, económica y fiscal.

El gobierno prometió públicamente realizar con firmeza varios cambios:

1. El saneamiento definitivo de las finanzas de los bancos.

2. Reforma estructural en todos los campos: principalmente en la nueva industria "tecnología de la información", obras públicas, seguridad social y las finanzas de las administraciones locales. 
3. Reactivación de la economía impulsando la iniciativa de la empresa privada.

Koizumi dejó claro que llevaría a cabo las reformas en todos los campos sin excepción, y que sin duda haría falta realizar sacrificios. La "Reforma Koizumi" promulgaba tres lemas:

a) No puede haber crecimiento sin reformas, y éstas implican sacrificios.

b) Hay que dejar en manos del sector privado aquello que pueda hacer.

c) Hay que dejar en manos de las administraciones locales los que éstas pueden realizar. ${ }^{5}$

Siete son los programas para las reformas estructurales:

1) La privatización y la desregulación

2) El apoyo a proyectos nuevos

3) La consolidación del funcionamiento de la seguridad social

4) El fortalecimiento de los bienes intelectuales

5) La innovación en el estilo de vida

6) La activación e independencia de las regiones

7) La reforma fiscal.

El programa de reformas consiste en apoyar con fuerza la privatización de las principales corporaciones públicas, y de convertir los organismos de Correos y Telecomunicaciones en corporaciones públicas como un primer paso a su privatización. En relación con esto a finales de 2001, el gobierno decidió privatizar cuatro corporaciones públicas relacionadas con las carreteras, empezando por la Corporación Pública de Autopistas de Japón, además de la Corporación Pública de Viviendas y Urbanización y la Corporación Pública del Petróleo.

También se ha impulsado la creación de centros competentes en el ámbito internacional creando universidades estatales gestionadas como las privadas, sin descartar su privatización. El objetivo de esta reforma de las universidades es convertir cuanto antes a las universidades estatales en corporaciones que puedan competir en el marco internacional y potenciar su capacidad en investigación y educación. Esta estrategia se propone impulsar, en los niveles de educación obligatoria, el sistema de libre elección de escuelas por parte de los alumnos, la promoción de la tecnología de la información en la educación y generalizar el aprendizaje del inglés.

El programa de apoyo a los proyectos innovadores se propone los siguiente:

- Poner en marcha nuevos negocios.

- Introducir un sistema tributario que propicie la creación de nuevas empresas.

- Reestructuración y renovación de las pequeñas y medianas empresas.

- Impulsar la revolución de la tecnología de la información y la educación en este campo.

En cuanto al programa para consolidar el funcionamiento de la seguridad social, se establece uno nuevo más "fiable", mediante la introducción de una contabilidad individual de la seguridad social (mayor control) y de la creación de un programa eficaz de seguro médico.

El programa para "incrementar los bienes intelectuales" fomenta el traspaso de fondos privados a la investigación y a la educación, dando prioridad estratégica a los sectores punteros como la nanotecnología, la TI, la biotecnología, el medio ambiente, etcétera. Todo basado en una estrategia para fortalecer la tecnología japonesa y poder competir con los países europeos y Estados Unidos.

El programa para innovar el estilo de vida se propone impulsar una ciudad de rascacielos multifuncionales, una mayor participación social de las mujeres, así como la creación de una sociedad sin desperdicios, ni efectos invernaderos, y la garantía de la seguridad ciudadana y el orden público.

Para esto habrá que superar varios obstáculos legales, llevando a cabo una reforma de las regulaciones. En lugar de permitir que todo el país se derrumbe por el peso de las 
regulaciones impuestas uniformemente, sería prudente una audaz desregulación experimental en esas zonas especiales.

El séptimo programa es la reforma fiscal, que consiste en un plan de dos fases. En la primera, se aspira sanear el fisco, frenando la emisión de bonos del Estado en menos de 30 millones de millones de yenes; en la segunda fase, el objetivo es conseguir un equilibrio primario entre los ingresos y los gastos fiscales a mediano plazo y lograr así un superávit. Japón es el único país industrializado que viene aumentando constantemente los proyectos en obras públicas sufragados por emisiones de bonos del Estado.

En el área de la reforma fiscal, uno de los cambios por realizar es referente a los impuestos: conseguir la rebaja de los tipos impositivos lo máximo posible, como incentivo para las empresas asegurando las finanzas necesarias para estas reducciones fiscales mediante mecanismos que no sean bonos del Estado (por ejemplo, frenando el gasto público).

Pero lo nuevo en la política de Koizumi es brindar una perspectiva más amplia del futuro y hará hincapié en las reformas vinculadas a cambiar la estructura económica de una economía industrial a la del conocimiento. Realizar una reforma estructural completa implica la creación de nuevas industrias y de oportunidades de empleo, lo cual es realizable a partir de la tecnología de la información. Esto implica llevar adelante la desregulación en todos los ámbitos económicos y sociales sin excepción, precisamente para impulsar, según este criterio, el desarrollo de la tecnología de la información.

Con esta estrategia se aspira a convertir a Japón en un líder mundial en dicha área en cinco años. Koizumi planteó que debe realizarse la transición de una economía industrial a otra del conocimiento, que garantice la seguridad nacional y regional, así como la superación del persistente estancamiento económico que sufre la economía japonesa.

\section{Conclusiones}

1. Japón es el primer país central que después de haber vivido un período ejemplar de altas tasa de crecimiento económico, enfrenta un proceso largo de desaceleración económica a finales del siglo XX e inicios del siglo XXI. Algunos economistas coinciden que Japón se ha convertido "en el gran fracaso de la macroeconomía moderna”. Pero evidentemente no será el último país central que pase por ese proceso. El camino del desarrollo hacia una "economía del conocimiento", sobre la base de la tecnología de la información, puede ser válido, siempre que no se acuda a la vieja receta de la llamada "austeridad", que conduce a un mercado nacional muy restringido, sobre la base del desempleo, el crecimiento del sector informal, los bajos salarios, un bajo presupuesto para sanidad, la educación, la vivienda, en fin, la seguridad social.

2. El impacto del estancamiento en Japón sobre la economía mundial es importante, ya que Japón produce el $12 \%$ del PIB mundial y es el mayor acreedor internacional.

3. Algunos especialistas japoneses plantean que la recesión ha sido positiva en algunas cuestiones:

- Obliga a los empresarios a luchar por una economía real, ya que durante la segunda mitad de los ochenta, cuando se aceleró la burbuja financiera, la administración de las corporaciones se habían relajado y se habían dedicado a buscar ganancias rápidas y fáciles con la especulación financiera.

- Muchas familias aceptan de manera gustosa que algunos de sus miembros no tengan que trabajar horas extras, porque así llegan más temprano a casa, aunque ganen menos. 
4. La crisis económica de Japón afecta los países de Asia. Como es el país líder de la región, controla el $40 \%$ del comercio con esa zona: el $30 \%$ de sus inversiones son en Asia y es el donante número uno de la Ayuda Oficial para el Desarrollo en la región.

La economía japonesa está sufriendo una serie de cambios que pensamos deben ser objeto de futuras investigaciones. Los japoneses se han caracterizado por tener una gran capacidad de ajuste en períodos relativamente cortos. Hoy se ha roto ese principio. Esperemos que sepan salir de esta crisis en el mediano plazo.

\section{Bibliografía}

Banco de Japón (2003) "Recent Trends in Business Fixed Investment and the Issues Attending a Full Recovery: Restoring Firm's Capacity to Generate Capital Investment", Quarterly Bulletin, noviembre.

Cuadernos de Japón, volumen XII, número 1, invierno 1999, pág. 45; volumen XV, número 3, 2002, pág. 4; volumen XV, número 3, 2002, pág. 6 .

"Crecimiento cero en economía japonesa en los primeros meses 2003”. Cable noticioso en Agencia Internacional Latinoamericana Prensa Latina S.A. ( corresponsal Tokio) 16 de Mayo del 2003
Oviedo, Luis . "Japón: la depresión económica y la economía mundial". En defensa del marxismo: revista teórica del partido obrero. Sumario del número 25. Diciembre 1999. [en línea] < http//www.po.org.ar/edm/edm25.htm> [ consultado 10 de julio 2003]

Rodríguez, Ernesché (1999) La economía de burbuja en Japón. Editorial Ciencias Sociales. Calle 14 no. 4104, Playa, Ciudad de la Habana, Cuba. Impreso en Editorial Linotipia Bolívar, Bogotá-Colombia. 106 p.

\section{Notas}

1 Nakatani Iwao. "Cinco puntos para evitar la quiebra", Cuadernos de Japón, volumen XII, número 1, invierno 1999, pág. 45.

2 Oviedo, Luis. “Japón: la depresión económica y la economía mundial”. En defensa del marxismo: revista teórica del partido obrero. Sumario del número 25. Diciembre 1999. [en línea] < http//www.po.org.ar/edm/ edm25.htm> [ consultado 10 de julio 2003]

3 Takenaka, Heizó. "Adelante con el programa de reformas”, Cuadernos de Japón, volumen XV, número 3, 2002, pág. 6 .

4 "Crecimiento cero en economía japonesa en los primeros meses 2003”. Cable noticioso en Agencia Internacional Latinoamericana Prensa Latina S.A. (corresponsal Tokio) 16 de mayo del 2003.

5 Takenaka, Heizó. "Adelante con el programa de reformas"Cuadernos de Japón, volumen XV, número 3, 2002, pág. 4. T:! 